\title{
NEW COMBINATORIAL INTERPRETATIONS OF TWO ANALYTIC IDENTITIES
}

\author{
A. K. AGARWAL
}

(Communicated by William Adams)

\begin{abstract}
Two generalized partition theorems involving partitions with " $n+$ 1 copies of $n$ " and " $n+2$ copies of $n$ ", respectively, are proved. These theorems have potential of yielding infinite Rogers-Ramanujan type identities on MacMahon's lines. Five particular cases are also discussed. Among them three are known and two provide new combinatorial interpretations of two known $q$-identities.
\end{abstract}

\section{INTRODUCTION, DEFINITIONS, AND MAIN RESULTS}

In this paper we shall prove some partition identities involving partitions with " $n+\ell$ copies on $n$ ". We first recall the following definitions from [3]:

Definition 1. A partition with " $n+\ell$ copies of $n$ ", $\ell \geq 0$ is a partition in which a part of size $n, n \geq 0$, can come in $n+\ell$ different colors denoted by subscripts: $n_{1}, n_{2}, \ldots, n_{n+\ell}$. In the part $n_{i}, n$ can be zero if and only if $i \geq 1$. But in no partition are zeros permitted to repeat.

Definition 2. The weighted difference of two parts $m_{i}, n_{j}, m \geq n$ is defined by $m-n-i-j$ and is denoted by $\left(\left(m_{i}-n_{j}\right)\right)$.

Recently in [1] the following result was proved:

Theorem 1. For $k \geq-3$, let $A_{k}(\nu)$ denote the number of partitions of $\nu$ with " $n$ copies of $n$ " such that the weighted difference of each pair of summands $m_{i}$, $n_{j}$ is greater than $k$. Then

$$
\sum_{\nu=0}^{\infty} A_{k}(\nu) q^{\nu}=\sum_{\nu=0}^{\infty} \frac{q^{\nu\left[1+\frac{(k+3)(\nu-1)}{2}\right]}}{(q ; q)_{\nu}\left(q ; q^{2}\right)_{\nu}}
$$

Received by the editors August 22, 1988.

1980 Mathematics Subject Classification (1985 Revision). Primary 05A15, 05A17, 05A19, 11 P57.

Key words and phrases. Partitions, weighted differences, $q$-identities, combinatorial interpretations. 
where

$$
(a ; q)_{n}=\prod_{i=0}^{\infty} \frac{\left(1-a q^{i}\right)}{\left(1-a q^{n+i}\right)} .
$$

Some particular cases were also discussed. Among them was the following identity in which the partitions enjoy a convolution property:

Corollary 1. $A_{-2}(\nu)$ equals $\sum_{k=0}^{\nu} A_{\nu-k} B_{k}$, where $A_{\nu}$ denotes the number of partitions of $\nu$ into distinct parts $\equiv \pm 3(\bmod 7)$ and $B_{\nu}$ denotes the number of partitions of $\nu$ into parts $\not \equiv 0, \pm 4 \quad(\bmod 14)$.

Corollary 1 is a combinatorial interpretation of the identity [5, Eq. (3.1), p. 219]:

$$
\sum_{n=0}^{\infty} \frac{q^{\frac{1}{2}\left(n^{2}+n\right)}}{(q ; q)_{n}\left(q ; q^{2}\right)_{n}}=\prod_{n=1}^{\infty} \frac{\left(1+q^{n}\right)\left(1-q^{7 n-2}\right)\left(1-q^{7 n-5}\right)\left(1-q^{7 n}\right)}{\left(1-q^{n}\right)\left(1+q^{7 n-1}\right)\left(1+q^{7 n-6}\right)}
$$

In this paper we shall prove the following two theorems, which are very similar to Theorem 1:

Theorem 2. For $k \geq-3$, let $B_{k}(\nu)$ denote the number of partitions of $\nu$ with " $n+1$ copies of $n$ " such that the weighted difference of each pair of parts is greater than $k$, the parts are nonnegative, and for some $i, i_{i+1}$ is a part. Then

$$
\sum_{\nu=0}^{\infty} B_{k}(\nu) q^{\nu}=\sum_{\nu=0}^{\infty} \frac{q^{\nu(\nu+1)(k+3) / 2}}{(q ; q)_{\nu}\left(q ; q^{2}\right)_{\nu+1}}
$$

Theorem 3. For $k \geq-3$, let $C_{k}(\nu)$ denote the number of partitions of $\nu$ with " $n+2$ copies of $n$ " into nonnegative parts such that the weighted difference of each pair of parts is greater than $k$, and for some $i, i_{i+2}$ is a part. Then

$$
\sum_{\nu=0}^{\infty} C_{k}(\nu) q^{\nu}=\sum_{\nu=0}^{\infty} \frac{q^{\nu[1+(\nu+1)(k+3) / 2]}}{(q ; q)_{\nu}\left(q ; q^{2}\right)_{\nu+1}} .
$$

In $\S 3$ we shall show that Theorem 2 and 3 provide new combinatorial interpretations (similar to Corollary 1 above) of the following $q$-identities [6, p. 160 $\mathrm{I}(80)$ and $\mathrm{I}(82)$, respectively]:

$$
\begin{aligned}
& \sum_{n=0}^{\infty} \frac{q^{\frac{1}{2} n(n+1)}}{(q ; q)_{n}\left(q ; q^{2}\right)_{n+1}} \\
& \quad=\frac{(-q ; q)_{\infty}}{(q ; q)_{\infty}} \prod_{n=1}^{\infty}\left(1-q^{7 n-2}\right)\left(1-q^{14 n-11}\right)\left(1-q^{14 n-3}\right)\left(1-q^{7 n}\right)
\end{aligned}
$$

and

$$
\begin{aligned}
& \sum_{n=0}^{\infty} \frac{q^{\frac{1}{2} n(n+3)}}{(q ; q)_{n}\left(q ; q^{2}\right)_{n+1}} \\
& =\frac{(-q ; q)_{\infty}}{(q ; q)_{\infty}} \prod_{n=1}^{\infty}\left(1-q^{7 n-3}\right)\left(1-q^{7 n-4}\right)\left(1-q^{14 n-13}\right)\left(1-q^{14 n-1}\right)\left(1-q^{7 n}\right) .
\end{aligned}
$$

In $\S 3$ we shall pose some very significant open problems. 


\section{Proofs of Theorems 2 AND 3}

Proof of Theorem 2. Let $A_{k}(m, \nu)$ and $B_{k}(m, \nu)$ denote respectively the number of partitions of $\nu$ enumerated by $A_{k}(\nu)$ and $B_{k}(\nu)$ with the added restriction that there be exactly $m$ parts.

It was shown in [1, Eq. (2.6)] that if

$$
f_{k}(z, q)=\sum_{m=0}^{\infty} \sum_{\nu=0}^{\infty} A_{k}(m, \nu) z^{m} q^{\nu}
$$

then

$$
f_{k}(z, q)=\sum_{\nu=0}^{\infty} \frac{q^{\nu[1+(k+3)(\nu-1) / 2]} z^{\nu}}{(q ; q)_{\nu}\left(q ; q^{2}\right)_{\nu}}
$$

and so

$$
f_{k}(z, q)-f_{k}(z q, q)=z q \sum_{\nu=0}^{\infty} \frac{q^{\nu(\nu+1)(k+3) / 2}(z q)^{\nu}}{(q ; q)_{\nu}\left(q ; q^{2}\right)_{\nu+1}}
$$

Setting

$$
\begin{aligned}
g_{k}(z, q) & =\sum_{\nu=0}^{\infty} \frac{q^{\nu(\nu+1)(k+3) / 2} z^{\nu}}{(q ; q)_{\nu}\left(q ; q^{2}\right)_{\nu+1}} \\
& =\sum_{m=0}^{\infty} \sum_{\nu=0}^{\infty} E_{k}(m, \nu) z^{m} q^{\nu},
\end{aligned}
$$

we see by coefficient comparision in (2.3) that

$$
A_{k}(m, \nu)-A_{k}(m, \nu-m)=E_{k}(m-1, \nu-m) .
$$

Equation (2.5) shows that $E_{k}(m, \nu)$ equals the number of partitions of $\nu+m+1$ with " $n$ copies $n$ " into $m+1$ parts such that the weighted difference of each pair of parts $m_{i}, n_{j}$ is greater than $k$ and, for some $i, i_{i}$ is a part. If we subtract 1 from each part of a partition enumerated by $E_{k}(m, \nu)$ ignoring the subscripts we see that the resulting partition is enumerated by $B_{k}(m+1, \nu)$. This implies that

$$
E_{k}(m, \nu)=B_{k}(m+1, \nu) .
$$

Hence

$$
\sum_{m=0}^{\infty} \sum_{\nu=0}^{\infty} B_{k}(m+1, \nu) z^{m} q^{\nu}=\sum_{m=0}^{\infty} \sum_{\nu=0}^{\infty} \frac{q^{\nu(\nu+1)(k+3) / 2} z^{m}}{(q ; q)_{\nu}\left(q ; q^{2}\right)_{\nu+1}}
$$

Now

$$
\sum_{\nu=0}^{\infty} B_{k}(\nu) q^{\nu}=\sum_{\nu=0}^{\infty}\left[\sum_{m=0}^{\infty} B_{k}(m, \nu)\right] q^{n}=g_{k}(1, q)=\sum_{\nu=0}^{\infty} \frac{q^{\nu(\nu+1)(k+3) / 2}}{(q ; q)_{\nu}\left(q ; q^{2}\right)_{\nu+1}} .
$$

This completes the proof of Theorem 2 . 
Proof of Theorem 3. Let $C_{k}(m, \nu)$ denote the number of partitions of $\nu$ counted by $C_{k}(\nu)$ with the added restriction that there be exactly $m$ parts. Equation (2.3) can be written as

$$
f_{k}(z, q)-f_{k}(z q, q)=z q h_{k}(z, q)
$$

where

$$
h_{k}(z, q)=\sum_{\nu=0}^{\infty} \frac{q^{\nu[1+(\nu+1)(k+3) / 2]} z^{\nu}}{(q ; q)_{\nu}\left(q ; q^{2}\right)_{\nu+1}}
$$

Setting

$$
h_{k}(z, q)=\sum_{m=0}^{\infty} \sum_{\nu=0}^{\infty} F_{k}(m, \nu) z^{m} q^{\nu},
$$

we see by coefficient comparison in (2.8) that

$$
A_{k}(m, \nu)-A_{k}(m, \nu-m)=F_{k}(m-1, \nu-1) .
$$

Equation (2.11) shows that $F_{k}(m, \nu)$ equals the number of partitions of $\nu+1$ with " $n$ copies of $n$ " into $m+1$ parts such that the weighted difference of each pair of parts $m_{i}, n_{j}$ is greater than $k$ and for some $i, i_{i}$ is a part. If we replace this part $i_{i}$ by $(i-1)_{i+1}$, we see that the resulting partition is enumerated by $C_{k}(m+1, \nu)$. This implies that

$$
F_{k}(m, \nu)=C_{k}(m+1, \nu) \text {. }
$$

Hence

$$
\sum_{m=0}^{\infty} \sum_{\nu=0}^{\infty} C_{k}(m+1, \nu) z^{m} q^{\nu}=\sum_{m=0}^{\infty} \sum_{\nu=0}^{\infty} \frac{q^{\nu[1+(\nu+1)(k+3) / 2]} z^{\nu}}{(q ; q)_{\nu}\left(q ; q^{2}\right)_{\nu+1}}
$$

Now

$$
\begin{aligned}
\sum_{\nu=0}^{\infty} c_{k}(\nu) q^{\nu} & =\sum_{\nu=0}^{\infty}\left\{\sum_{m=0}^{\infty} C_{k}(m, \nu)\right\} q^{\nu}=h_{k}(1, q) \\
& =\sum_{\nu=0}^{\infty} \frac{q^{\nu[1+(\nu+1)(k+3) / 2]}}{(q ; q)_{\nu}\left(q ; q^{2}\right)_{\nu+1}}
\end{aligned}
$$

and the proof of Theorem 3 is completed.

\section{Particular cases}

For $k=0$, Theorem 2 , in view of the identity $[6, \mathrm{I}(4)$, p. 156]

$$
\sum_{n=0}^{\infty} \frac{q^{3 n(n+1) / 2}}{(q ; q)_{n}\left(q ; q^{2}\right)_{n}}=\frac{1}{(q ; q)_{\infty}} \prod_{n=1}^{\infty}\left(1-q^{10 n}\right)\left(1-q^{10 n-2}\right)\left(1-q^{10 n-8}\right)
$$

yields 
Corollary 2.1. The number of partitions of $\nu$ with " $n+1$ copies of $n$ " into nonnegative parts, such that each pair of parts $m_{i}, n_{j}$ has positive weighted difference and for some $i, i_{i+1}$ is a part, equals the number of ordinary partitions of $\nu$ into parts $\not \equiv 0, \pm 2 \quad(\bmod 10)$.

For $k=-1$, Theorem 2 , in view of the identity $[6, \mathrm{I}(60)$, p. 158]

$$
\sum_{n=0}^{\infty} \frac{q^{n(n+1)}}{(q ; q)_{n}\left(q ; q^{2}\right)_{n+1}}=\frac{1}{(q ; q)_{\infty}} \prod_{n=1}^{\infty}\left(1-q^{14 n-4}\right)\left(1-q^{14 n-10}\right)\left(1-q^{14 n}\right)
$$

reduces to

Corollary 2.2. The number of partitions of $\nu$ with " $n+1$ copies of $n$ " into nonnegative parts, such that each pair of parts $m_{i}, n_{j}$ has nonnegative weighted difference and for some $i, i_{i+1}$ is a part, equals the number of ordinary partitions of $\nu$ into parts $\not \equiv 0, \pm 4(\bmod 14)$.

On the other hand, in this particular case, Theorem 3, in view of the identity $[6, \mathrm{I}(59)$, p. 157]

$$
\sum_{n=0}^{\infty} \frac{q^{n(n+2)}}{(q ; q)_{n}\left(q ; q^{2}\right)_{n+1}}=\frac{1}{(q ; q)_{n}} \prod_{n=1}^{\infty}\left(1-q^{14 n-2}\right)\left(1-q^{14 n-12}\right)\left(1-q^{14 n}\right),
$$

reduces to

Corollary 3.1. The number of partitions of $\nu$ with " $n+2$ copies of $n$ " into nonnegative parts, such that each pair of parts $m_{i}, n_{j}$ has nonnegative weighted difference and for some $i, i_{i+2}$ is a part, equals the number of ordinary partitions of $\nu$ into parts $\not \equiv 0, \pm 2(\bmod 14)$.

Remark 1. Corollaries 2.1, 2.2, and 3.1 are the particular cases $k=2, \ell=1$; $k=3, \ell=1$; and $k=3, \ell=2$, respectively of Theorem 4 in [3].

Remark 2. Another proof of Corollaries 2.1, 2.2, and 3.1 can be found in [4]. For $k=-2$, Theorems 2 and 3 give the following combinatorial interpretations, presumably new, of the identities (1.5) and (1.6), respectively.

Corollary 2.3. The number of partitions of $\nu$ with " $n+1$ copies of $n$ " into nonnegative parts, such that the weighted difference of each pair of summands $m_{i}, n_{j}$ is greater than or equal to -1 and for some $i, i_{i+1}$ is a part, equals $\sum_{k=0}^{\nu} C_{k} D_{\nu-k}$, where $C_{k}$ denotes the number of partitions $k$ into parts $\equiv \pm 1, \pm 4, \pm 6 \quad(\bmod 14)$ and $D_{k}$ denotes the number of partitions of $k$ into distinct parts.

Example. $B_{-2}(5)=12$, since the relevant partitions are $5_{6}, 5_{1}+0_{1}, 5_{2}+0_{1}$, $5_{3}+0_{1}, 5_{4}+0_{1}, 5_{5}+0_{1}, 4_{1}+1_{2}, 4_{2}+1_{2}, 4_{1}+1_{1}+0_{1}, 4_{2}+1_{1}+0_{1}, 4_{3}+1_{1}+0_{1}$, $3_{1}+2_{1}+0_{1}$. 
Also,

$$
\begin{aligned}
\sum_{k=0}^{5} C_{k} D_{5-k} & =C_{0} D_{5}+C_{1} D_{4}+C_{2} D_{3}+C_{3} D_{2}+C_{4} D_{1}+C_{5} D_{0} \\
& =1(3)+1(2)+1(2)+1(1)+2(1)+2(1) \\
& =12 .
\end{aligned}
$$

Corollary 3.2. The number of partitions of $\nu$ with " $n+2$ copies of $n$ " into nonnegative parts, such that the weighted difference of each pair of summands $m_{i}, n_{j}$ is greater than or equal to -1 and for some $i, i_{i+2}$ is a part, equals $\sum_{k=0}^{\nu} E_{k} D_{\nu-k}$, where $E_{k}$ denotes the number of partitions of $k$ into parts $\equiv$ $2,5,6,8,9,12(\bmod 14)$ and $D_{k}$, as in Corollary 2.3, denotes the number of partitions of $k$ into distinct parts.

Example. $C_{-2}(6)=10$, since the relevant partitions are $6_{8}, 6_{1}+0_{2}, 6_{2}+0_{2}$, $6_{3}+0_{2}, 6_{4}+0_{2}, 6_{5}+0_{2}, 4_{1}+2_{1}+0_{2}, 4_{2}+2_{1}+0_{2}, 5_{1}+1_{3}, 5_{2}+1_{3}$. Also,

$$
\begin{aligned}
\sum_{\nu=0}^{6} E_{k} D_{6-k} & =E_{0} D_{6}+E_{1} D_{5}+E_{2} D_{4}+E_{3} D_{3}+E_{4} D_{2}+E_{5} D_{1}+E_{6} D_{0} \\
& =1(4)+0(3)+1(2)+0(2)+1(1)+1(1)+2(1) \\
& =10 .
\end{aligned}
$$

\section{Conclusion}

Many questions arise from this work. The most obvious among them are:

(1) Can Theorems 1, 2, and 3 be combined into one?

(2) Is it possible to give a nice combinatorial interpretation of Theorems 1 , 2 , and 3 for the general value of $k$ ?

(3) The methods used here were also used recently in [2] to give $n$-color partitions theoretic interpretations of several $q$-identities from [6]. Is it possible to prove Theorems 3 and 4 of [3] by using these methods?

(4) We have seen that each of Theorems 1, 2, and 3 yields a partition identity wherein the partitions enjoy a convolution property. Does this lead to the conclusion that there exists an infinite family of identities wherein partitions enjoy a convolution property?

\section{REFERENCES}

1. A. K. Agarwal, Partitions with " $N$ copies of $N$ ", Lecture Notes in Math., no. 1234, Proceedings of the Colloque de Combinatorie Énumerative, University of Quebec at Montreal, Springer-Verlag, 1985, pp. 1-4.

2. __ Rogers-Ramanujan identities for $n$-color partitions, J. Number Theory 28 (1988), 299-305

3. A. K. Agarwal and G. E. Andrews, Rogers-Ramanujan identities for partitions with " $N$ copies of $N$, J. Combin. Theory Ser. A, 45, No. 1 (1987), 40-49. 
4. A. K. Agarwal and D. M. Bressoud, Lattice paths and multiple basic hypergeometric series, Pacific J. Math., 136, No. 2 (1989), 209-228.

5. W. N. Bailey, On the simplification of some identities of the Rogers-Ramanugan type, Proc. London Math. Soc. (3) 1 (1951), 217-221.

6. L. J. Slater, Further identities of the Rogers-Ramanugan type, Proc. London Math. Soc. 54 (1951-52), 147-167.

Department of Mathematics, Pennsylvania State University, Mont Alto, PENNSYLVANIA 17237 